\title{
A DIOPHANTINE PROBLEM FOR LAURENT POLYNOMIAL RINGS
}

\author{
PETER PAPPAS
}

\begin{abstract}
Let $R$ be an integral domain of characteristic zero. We prove that the diophantine problem for the Laurent polynomial ring $R\left[T, T^{-1}\right]$ with coefficients in $\mathbf{Z}[T]$ is unsolvable. Under suitable conditions on $R$ we then show that either $\mathbf{Z}$ or $\mathbf{Z}[i]$ is diophantine over $R\left[T, T^{-1}\right]$.
\end{abstract}

1. Introduction. Let $R$ be a commutative ring with identity and let $S$ be a fixed recursive subring of $R$, i.e. there exists a bijective map $\theta: \mathbf{N} \rightarrow S$ such that the pre-images of the ring operations are recursive in $\mathbf{N}$ (see, e.g., Rabin [5]). The diophantine problem for $R$ with coefficients in $S$ is said to be unsolvable (solvable) if there exists no (an) algorithm to decide whether or not a diophantine equation in several variables with coefficients in $S$ has a solution in $R$. The diophantine problem for the complex function field $\mathbf{C}(T)$ with coefficients in $\mathbf{Z}[T]$ is very much an open question. Related results are as follows:

TheOREM A (DeneF [3]). Let $R$ be an integral domain of characteristic zero. Then the diophantine problem for $R[T]$ with coefficients in $\mathbf{Z}[T]$ is unsolvable.

TheOREM B (DENEF [3]). Let $K$ be a formally real field. Then the diophantine problem for $K(T)$ with coefficients in $\mathbf{Z}[T]$ is unsolvable.

Now let $R$ be an integral domain of characteristic zero with quotient field $F$. The smallest ring containing $R$ in which $T$ is invertible is the Laurent polynomial ring $R\left[T, T^{-1}\right] \subset F(T)$.

Our main theorem is the following:

THEOREM. Let $R$ be an integral domain of characteristic zero. Then the diophantine problem for $R\left[T, T^{-1}\right]$ with coefficients in $\mathbf{Z}[T]$ is unsolvable.

In light of Theorem A our result is not surprising; in fact, our proof follows the same route. However, there is an interesting difference. We need to consider two cases, namely $\sqrt{-1} \notin R$ and $\sqrt{-1} \in R$.

To handle the first case we use the well-known result of M. Davis, Yu. Matijasevic, H. Putnam and J. Robinson (see, e.g., [1]) that Hilbert's tenth problem is unsolvable, and for the second case we rely on a result of Denef which states that the diophantine problem for the ring of Gaussian integers $\mathbf{Z}[i]$ with coefficients in $\mathbf{Z}$ is unsolvable (see [2] or his generalization in [4]).

Received by the editors March 30, 1984.

1980 Mathematics Subject Classification. Primary 03B25, 10 N05.

Key words and phrases. Diophantine problems, unsolvable problems, Hilbert's tenth problem.

1985 American Mathematical Society $0002-9939 / 85 \$ 1.00+\$ .25$ per page 
2. The solution. We shall stay with the notation from [3] and begin by setting up some general terminology. Let $D\left(x_{1}, \ldots, x_{n}\right)$ be a relation on $R\left[T, T^{-1}\right]$. We say that $D\left(x_{1}, \ldots, x_{n}\right)$ is diophantine over $R\left[T, T^{-1}\right]$ with coefficients in $\mathbf{Z}[T]$ if there exists a diophantine equation $P\left(x_{1}, \ldots, x_{n}, y_{1}, \ldots, y_{m}\right)$ over $\mathbf{Z}[T]$ such that, for all $x_{1}, \ldots, x_{n}$ $\in R\left[T, T^{-1}\right]$ :

$$
D\left(x_{1}, \ldots, x_{n}\right) \leftrightarrow \exists y_{1}, \ldots, y_{m} \in R\left[T, T^{-1}\right]: P\left(x_{1}, \ldots, x_{n}, y_{1}, \ldots, y_{m}\right)=0 .
$$

We note that if $D_{1}$ and $D_{2}$ are diophantine over $R\left[T, T^{-1}\right]$ with coefficients in $\mathrm{Z}[T]$, then so are $D_{1} \vee D_{2}$ and $D_{1} \wedge D_{2}$. Indeed, $P_{1}=0 \vee P_{2}=0 \leftrightarrow P_{1} P_{2}=0$ and $P_{1}=0 \wedge P_{2}=0 \leftrightarrow P_{1}^{2}+T P_{2}^{2}=0$.

Consider the Pell equation

$$
X^{2}-\left(T^{2}-1\right) Y^{2}=1
$$

and let $U$ be an element in the algebraic closure of $R\left[T, T^{-1}\right]$ satisfying

$$
U^{2}=T^{2}-1 \text {. }
$$

Then we have

$$
(X+U Y)(X-U Y)=1 \text {. }
$$

Let $X, Y \in R\left[T, T^{-1}\right]$ satisfy (1). As an algebraic function of $T, X+U Y$ can be written in the form

$$
g(T) / T^{r}+\sqrt{T^{2}-1} f(T) / T^{s}
$$

with $g(T), f(T) \in R[T]$ and $r, s \in \mathbf{N}$. We next parametrize the curve (2) by

$$
T=t^{2}+1 / t^{2}-1, \quad U=2 t / t^{2}-1 \text {. }
$$

As rational functions of $t$, it is easily seen that $X+U Y$ and $X-U Y$ have poles only at $t= \pm 1, \pm i$. Furthermore, (3) implies that they have zeros only at $t= \pm 1, \pm i$.

Now observe that $(X+U Y)(-t)=(X-U Y)(t)$ and so we conclude that if $X, Y \in R\left[T, T^{-1}\right]$ is a solution of (1), then

$$
X+U Y=c\left(\frac{t-1}{t+1}\right)^{m}\left(\frac{t-i}{t+i}\right)^{n}, \quad X-U Y=c\left(\frac{t-1}{t+1}\right)^{-m}\left(\frac{t-i}{t+i}\right)^{-n},
$$

for some $c \in R$ and some $m, n \in \mathbf{Z}$. Substituting these two expressions into (3) yields $c^{2}=1$.

Let us now consider $X+U Y$ as an algebraic function of $T$ and suppose for the moment that $c=1$. (The case $c=-1$ is entirely similar to this one.) We have

$$
\begin{aligned}
X+U Y & =\left(\frac{t-1}{t+1}\right)^{m}\left(\frac{t-i}{t+i}\right)^{n} \\
& =(T+U)^{m}\left(\frac{t^{2}-1}{t^{2}+1}-i \frac{2 t}{t^{2}+1}\right)^{n}=(T+U)^{m}\left(\frac{1-i U}{T}\right)^{n} .
\end{aligned}
$$

From (2),

$$
(T-U)^{-m}=(T+U)^{m} \text { and }\left(\frac{1-i U}{T}\right)^{-n}=\left(\frac{1+i U}{T}\right)^{n},
$$


and therefore we may rewrite $X+U Y$ and $X-U Y$ as expressions involving only positive integral exponents.

Thus if $(X, Y) \in R\left[T, T^{-1}\right] \times R\left[T, T^{-1}\right]$ is a solution to (1), we have one of four possible outcomes, namely

$$
\begin{array}{ll}
X+U Y=(T+U)^{m}\left(\frac{1-i U}{T}\right)^{n}, & \\
X-U Y=(T-U)^{m}\left(\frac{1-i U}{T}\right)^{n}, & \text { some }(m, n) \in \mathbf{N} \times \mathbf{N} ; \\
X+U Y=(T+U)^{m}\left(\frac{1+i U}{T}\right)^{n}, & \text { some }(m, n) \in \mathbf{N} \times \mathbf{N} ; \\
X-U Y=(T-U)^{m}\left(\frac{1-i U}{T}\right)^{n}, & \\
X+U Y=(T-U)^{m}\left(\frac{1-i U}{T}\right)^{n}, & \text { some }(m, n) \in \mathbf{N} \times \mathbf{N} ; \\
X-U Y+(T+U)^{m}\left(\frac{1+i U}{T}\right)^{n}, & \\
X+U Y=(T-U)^{m}\left(\frac{1+i U}{T}\right)^{n}, & \text { some }(m, n) \in \mathbf{N} \times \mathbf{N} . \\
X-U Y=(T+U)^{m}\left(\frac{1-i U}{T}\right)^{n}, &
\end{array}
$$

Now let $S$ denote the ring $\mathbf{Z}[i]\left[T, T^{-1}\right]$. By (2), $S[U]$ defines a quadratic ring extension of $S$. For each $j=1,2,3,4$ define two sequences $X_{(m, n)}^{(j)}, Y_{(m, n)}^{(j)},(m, n) \in$ $\mathbf{N} \times \mathbf{N}$, of elements of $S$ by

$$
\begin{aligned}
& X_{(m, n)}^{(1)}+U Y_{(m, n)}^{(1)}=(T+U)^{m}\left(\frac{1-i U}{T}\right)^{n}, \\
& X_{(m, n)}^{(2)}+U Y_{(m, n)}^{(2)}=(T+U)^{m}\left(\frac{1+i U}{T}\right)^{n}, \\
& X_{(m, n)}^{(3)}+U Y_{(m, n)}^{(3)}=(T-U)^{m}\left(\frac{1-i U}{T}\right)^{n}, \\
& X_{(m, n)}^{(4)}+U Y_{(m, n)}^{(4)}=(T-U)^{m}\left(\frac{1+i U}{T}\right)^{n} .
\end{aligned}
$$

Applying the ring automorphism $S[U] \rightarrow S[U]$, which fixes $S$ elementwise and sends $U$ to $-U$, together with (2) yields

$$
\begin{aligned}
& X_{(m, n)}^{(1)}-U Y_{(m, n)}^{(1)}=(T-U)^{m}\left(\frac{1+i U}{T}\right)^{n}=(T+U)^{-m}\left(\frac{1-i U}{T}\right)^{-n}, \\
& X_{(m, n)}^{(2)}-U Y_{(m, n)}^{(2)}=(T-U)^{m}\left(\frac{1-i U}{T}\right)^{n}=(T+U)^{-m}\left(\frac{1-i U}{T}\right)^{-n}, \\
& X_{(m, n)}^{(3)}-U Y_{(m, n)}^{(3)}=(T+U)^{m}\left(\frac{1+i U}{T}\right)^{n}=(T-U)^{-m}\left(\frac{1-i U}{T}\right)^{-n}, \\
& X_{(m, n)}^{(4)}-U Y_{(m, n)}^{(4)}=(T+U)^{m}\left(\frac{1-i U}{T}\right)^{n}=(T-U)^{-m}\left(\frac{1+i U}{T}\right)^{-n},
\end{aligned}
$$


and hence, for every $(m, n) \in \mathbf{N} \times \mathbf{N}$ and each $j=1,2,3,4$, the pair $\left(X_{(m, n)}^{(j)}\right.$, $\left.Y_{(m, n)}^{(j)}\right) \in \mathbf{S} \times \mathbf{S}$ is a solution to (1).

LEMMA 1. (a) If $i \in R$, then the solutions of (1) in $R\left[T, T^{-1}\right]$ are of the form

$$
\left(X_{(m, n)}^{(j)}, Y_{(m, n)}^{(j)}\right), \quad(m, n) \in \mathbf{N} \times \mathbf{N}, j=1,2,3,4 .
$$

(b) If $i \notin R$, then the solutions of (1) in $R\left[T, T^{-1}\right]$ are of the form

$$
\left(X_{(m, 0)}^{(j)}, Y_{(m, 0)}^{(j)}\right), \quad m \in \mathbf{N}, j=1,2,3,4 .
$$

Proof. By the foregoing discussion, it remains only to show that if $i \notin R$, then for every $(m, n) \in \mathbf{N} \times \mathbf{N}^{>0}$ and $j=1,2,3,4$,

$$
\left(X_{(m, n)}^{(j)}, Y_{(m, n)}^{(j)}\right) \notin R\left[T, T^{-1}\right] \times R\left[T, T^{-1}\right] .
$$

Fix $x=X_{(m, n)}^{(j)}, y=Y_{(m, n)}^{(j)}$ for some $(m, n) \in \mathbf{N} \times \mathbf{N}^{>0}, j \in\{1,2,3,4\}$, and assume $(x, y) \in R\left[T, T^{-1}\right] \times R\left[T, T^{-1}\right]$. Let $\sigma: S[U] \rightarrow S[U]$ be the ring automorphism which fixes $T$ and $U$ and sends $i$ to $-i$. Then $\sigma(x+U y)=x+U y$, which by (4)-(7) implies

$$
\left(\frac{1+i U}{T}\right)^{n}=\left(\frac{1-i U}{T}\right)^{n}
$$

Since this is impossible for $n>0$, we obtain the desired contradiction, and the lemma is complete.

Definition. Write $V \sim W$ if the elements $V, W \in R\left[T, T^{-1}\right]$ take on the same value at $T=1$.

LEMMA 2. The relation $Z \sim 0$ is diophantine over $R\left[T, T^{-1}\right]$ with coefficients in $\mathbf{Z}[T]$.

Proof. $Z \sim 0 \Leftrightarrow \exists X \in R\left[T, T^{-1}\right]: Z=(T-1) X$.

Lemma 3. (a) If $i \in R$, then $\left\{Y(1):(X, Y) \in X^{2}-\left(T^{2}-1\right) Y^{2}=1, X, Y \in\right.$ $\left.R\left[T, T^{-1}\right]\right\}=\mathbf{Z}[i]$.

(b) If $i \notin R$, then $\left\{Y(1)\right.$ : $\left.(X, Y) \in X^{2}-\left(T^{2}-1\right) Y^{2}=1, X, Y \in R\left[T, T^{-1}\right]\right\}=\mathbf{Z}$.

Proof. We shall give the explicit form of $Y_{(m, n)}^{(j)}$. We begin by noting

$$
Y_{(m, n)}^{(j)}=\frac{\left(X_{(m, n)}^{(j)}+U Y_{(m, n)}^{(j)}\right)-\left(X_{(m, n)}^{(j)}-U Y_{(m, n)}^{(j)}\right)}{2 U},
$$

from which it follows that $Y_{(m, n)}^{(3)}=-Y_{(m, n)}^{(2)}$ and $Y_{(m, n)}^{(4)}=-Y_{(m, n)}^{(1)}$. Using the binomial theorem and (8), we have:

For $(m, n) \in \mathbf{N}^{>0} \times N^{>0}$,

$$
\begin{aligned}
T^{n} Y_{(m, n)}^{(1)}= & T^{n} Y_{(m, n)}^{(2)}=\left(\sum_{\substack{j=0 \\
j \text {-odd }}}^{m}\left(\begin{array}{c}
m \\
j
\end{array}\right) T^{m-j} U^{j-n}\right)\left(\sum_{\substack{j=0 \\
j \text {-even }}}^{n}\left(\begin{array}{c}
n \\
j
\end{array}\right)(i U)^{j}\right) \\
& -\left(\sum_{\substack{j=0 \\
j \text {-even }}}^{m}\left(\begin{array}{c}
m \\
j
\end{array}\right) T^{m-j} U^{j}\right)\left(\sum_{\substack{j=0 \\
j \text {-odd }}}^{n}\left(\begin{array}{c}
n \\
j
\end{array}\right)(i)^{j} U^{j-1}\right) .
\end{aligned}
$$


$\operatorname{For}(m, n) \in \mathbf{N} \times\{0\}$,

$$
Y_{(m, n)}^{(1)}=Y_{(m, n)}^{(2)}=\sum_{\substack{j=0 \\
j \text {-odd }}}^{m}\left(\begin{array}{c}
m \\
j
\end{array}\right) T^{m-j} U^{j-1}
$$

$\operatorname{For}(0, n) \in\{0\} \times \mathbf{N}$

$$
T^{n} Y_{(0, n)}^{(1)}=\sum_{\substack{j=0 \\
j \text {-odd }}}\left(\begin{array}{c}
n \\
j
\end{array}\right)(-i)^{j} U^{j-1}
$$

and

$$
T^{n} Y_{(0, n)}^{(2)}=\sum_{\substack{j=0 \\
j \text {-odd }}}\left(\begin{array}{l}
n \\
j
\end{array}\right)\left(i^{j}\right) U^{j-1} .
$$

Using (2) and Lemma 1, and setting $T=1$ and $c= \pm 1$ yields the desired result.

Definition. $\operatorname{Imt}(Y) \Leftrightarrow Y \in R\left[T, T^{-1}\right] \wedge \exists X \in R\left[T, T^{-1}\right]: X^{2}-\left(T^{2}-1\right) Y^{2}=$ 1 .

Notice that Imt is diophantine over $R\left[T, T^{-1}\right]$ with coefficients in $\mathbf{Z}[T]$.

Proof OF THE THEOREM.

Case (a). $i \in R$. There exists an algorithm to find for any diophantine equation $P \in \mathbf{Z}\left[X_{1}, \ldots, X_{N}\right]$ a diophantine equation $P^{*} \in \mathbf{Z}[T]\left[X_{1}, \ldots, X_{N}\right]$ satisfying

$$
\begin{aligned}
& \exists z_{1}, \ldots, z_{N} \in \mathbf{Z}[i]: P\left(z_{1}, \ldots, z_{N}\right)=0 \\
& \quad \leftrightarrow Z_{1}, \ldots, Z_{N} \in R\left[T, T^{-1}\right]: P^{*}\left(Z_{1}, \ldots, Z_{N}\right)=0 .
\end{aligned}
$$

Indeed, by Lemma 3 we have

$$
\begin{aligned}
& \exists z_{1}, \ldots, z_{N} \in \mathbf{Z}[i]: P\left(z_{1}, \ldots, z_{N}\right)=0 \\
& \leftrightarrow Z_{1}, \ldots, Z_{N} \in R\left[T, T^{-1}\right]:\left(\operatorname{Imt}\left(Z_{1}\right) \wedge \cdots \wedge \operatorname{Imt}\left(Z_{N}\right)\right) \wedge P\left(Z_{1}, \ldots, Z_{N}\right) \sim 0 .
\end{aligned}
$$

Since Imt and are diophantine over $R\left[T, T^{-1}\right]$ with coefficients in $\mathbf{Z}[T]$, (9) follows. Thus if the diophantine problem for $R\left[T, T^{-1}\right]$ with coefficients in $\mathbf{Z}[T]$ would be solvable, then so would the diophantine problem for $\mathbf{Z}[i]$ with coefficients in $\mathbf{Z}$, contradicting Denef's result in [2].

Case (b). $i \notin R$. In exactly the same way we see that if the diophantine problem for $R\left[T, T^{-1}\right]$ with coefficients in $\mathbf{Z}[T]$ would be solvable, then so would Hilbert's tenth problem.

As in [3], we obtain the following corollary.

Corollary. (a) Let $R$ be an integral domain of characteristic zero with $i \in R$. Suppose there exists a subset $S$ of $R$ which contains $\mathbf{Z}[i]$ and which is diophantine over $R\left[T, T^{-1}\right]$; then $\mathbf{Z}[i]$ is diophantine over $R\left[T, T^{-1}\right]$. In particular, this is true where $R$ contains $\mathbf{Q}(i)$.

(b) Let $R$ be an integral domain of characteristic zero with $i \notin R$. Suppose there exists a subset $S$ of $R$ which contains $\mathbf{Z}$ and which is diophantine over $R\left[T, T^{-1}\right]$; then $\mathbf{Z}$ is diophantine over $R\left[T, T^{-1}\right]$. In particular, this is true when $R$ contains $\mathbf{Q}$. 
Proof. We prove only (a) since (b) follows similarly. If $S$ satisfies the conditions of the corollary, then

$$
z \in \mathbf{Z}[i] \leftrightarrow \exists Z \in R\left[T, T^{-1}\right](\operatorname{Imt}(Z) \wedge Z \sim z \wedge \in S) .
$$

Moreover, if $R$ contains $\mathbf{Q}(i)$, then we define $S$ by

$$
\begin{aligned}
x \in S \leftrightarrow x & \in R\left[T, T^{-1}\right] \\
& \wedge\left(x=0 \vee x=1 \vee \exists y_{1}, y_{2} x y_{1}=1 \wedge(x-1) y_{2}=1\right) .
\end{aligned}
$$

\section{REFERENCES}

1. M. Davis, Hilbert's tenth problem is unsolvable, Amer. Math. Monthly 80 (1973), 233-269.

2. J. Denef, Hilbert's tenth problem for quadratic rings. Proc. Amer. Math. Soc. 48 (1975), 214-220.

3. The diophantine problem for polynomial rings and fields of rational functions, Trans. Amer. Math. Soc. 242 (1978), 391-399.

4. Diophantine sets over algebraic integer rings. II, Trans. Amer. Math. Soc. 257 (1980), 227-236.

5. M. O. Rabin, Computable algebra, general theory and theory of computable fields, Trans. Amer. Math. Soc. 95 (1960), 341-360.

Department of Mathematics, Vassar College, Poughkeepsie, New York 12601 